\title{
An empirical approach to the extragalactic background light from AEGIS galaxy SED-type fractions
}

\author{
Alberto Domínguez ${ }^{1,2,3}$ \\ ${ }^{1}$ UCO/Lick Observatory, Dept. of Astronomy \& Astrophysics, University of California, Santa \\ Cruz, CA 95064, USA \\ ${ }^{2}$ Dept. of Physics, University of California, Santa Cruz, CA 95064, USA \\ ${ }^{3}$ Now at: Dept. of Physics \& Astronomy, University of Caliornia, Riverside, CA 92521, USA
}

email: alberto.dominguez@ucr.edu

\begin{abstract}
The extragalactic background light (EBL) is of fundamental importance both for understanding the entire process of galaxy evolution and for $\gamma$-ray astronomy. However, the overall spectrum of the EBL between 0.1 and $1000 \mu \mathrm{m}$ has never been determined directly, neither from observed luminosity functions (LFs), over a wide redshift range, nor from any multiwavelength observation of galaxy spectral energy distributions (SEDs). The evolving overall spectrum of the EBL is derived here utilizing a novel method based on observations only. It is emphasized that the local EBL seems already well constrained from the UV up to the mid-IR. Different independent methodologies such as direct measurement, galaxy counts, $\gamma$-ray attenuation and realistic EBL modelings point towards the same EBL intensity level. Therefore, a relevant contribution from Pop III stars to the local EBL seems unlikely.
\end{abstract}

Keywords. galaxies: formation, galaxies: evolution, cosmology: observations - diffuse radiation

\section{Introduction}

The extragalactic background light (EBL) is the accumulated radiation in the Universe from the star formation process, plus a contribution from active galactic nuclei (AGNs). These photons mostly lie in the range $\sim 0.1-1000 \mu \mathrm{m}$. The direct measurement of the EBL is a very difficult task subject to high uncertainties. This is mainly due to the contribution of zodiacal light, some orders of magnitude larger than the EBL (e.g., Hauser \& Dwek 2001; Chary \& Pope 2010). Interestingly, Matsuoka et al. (2011) have recently claimed a detection of the EBL free of zodiacal light. Other observational approaches set reliable lower limits on the EBL, such as measuring the integrated light from discrete extragalactic sources (e.g., Madau \& Pozzetti 2000; Fazio et al. 2004; Keenan et al. 2010). On the other hand, there are phenomenological approaches in the literature that predict an overall EBL model (i.e., between 0.1 and $1000 \mu \mathrm{m}$ and for any redshift). These are basically of the four kinds described in Domínguez et al. (2011a) and enumerated here in Table 1. Generally, any EBL modeling is built from two main quantities: one describing the galaxy density evolution over time and another one describing the overall galaxy emission (stellar component plus absorption/re-emission by dust). Table 1 briefly summarizes how these two main quantities are treated in the most relevant EBL modelings in the bibliography. We stress that the previous four-type classification is based upon how the different methodologies describe the galaxy density evolution.

We consider the theoretical approach taken in Somerville et al. (2011) and Gilmore et al. (2011) as complementary to our observationally motivated one to eventually reach 


\begin{tabular}{|c|c|c|}
\hline Type of modeling \& refs. & Galaxy density evolution & Galaxy emission \\
\hline $\begin{array}{l}\text { (i) Forward evolution, } \\
\text { e.g., Somerville et al. } \\
\text { (2011), Gilmore et al. } \\
\text { (2011) }\end{array}$ & $\begin{array}{l}\text { Semi-analytical mod- } \\
\text { els }\end{array}$ & $\begin{array}{l}\text { Modeled. Stellar emission: } \\
\text { Bruzual \& Charlot (2003) (BC03); } \\
\text { Dust absorption: Charlot \& Fall } \\
\text { (2000); Dust re-emission: templates } \\
\text { by Rieke et al. }(2009)\end{array}$ \\
\hline $\begin{array}{l}\text { (ii) Backward evolution, } \\
\text { e.g., Franceschini et al. } \\
(2008)\end{array}$ & $\begin{array}{l}\text { Observed local-optical } \\
\text { galaxy luminosity func- } \\
\text { tion (LF, for starburst } \\
\text { population) and near-IR } \\
\text { galaxy LF observed up } \\
\text { to } z=1.4 \text { (for elliptical } \\
\text { and spiral populations) }\end{array}$ & $\begin{array}{l}\text { Modeled. A few galaxy types mor- } \\
\text { phologically classified based on op- } \\
\text { tical images. }\end{array}$ \\
\hline $\begin{array}{l}\text { (iii) Inferred evolution, } \\
\text { e.g., Finke et al. (2010), } \\
\text { Kneiske \& Dole (2010) }\end{array}$ & $\begin{array}{l}\text { Parameterization of } \\
\text { the history of the star } \\
\text { formation rate density } \\
\text { of the Universe }\end{array}$ & $\begin{array}{l}\text { Modeled. Stellar emission: Sin- } \\
\text { gle bursts of solar metallicity from } \\
\text { Bruzual \& Charlot (1993) (in } \\
\text { Kneiske \& Dole 2010)/BC03 (in } \\
\text { Finke et al. 2010); Dust absorp- } \\
\text { tion: General extinction law; Dust } \\
\text { re-emission: Modified black bodies. } \\
\text { AGN galaxies are not considered. }\end{array}$ \\
\hline $\begin{array}{l}\text { (iv) Observed evolution, } \\
\text { Domínguez et al. (2011a) }\end{array}$ & $\begin{array}{l}\text { Observed near-IR } \\
\text { galaxy LF up to } z=4\end{array}$ & $\begin{array}{l}\text { Observed. Based on multiwave- } \\
\text { length photometry from the UV up } \\
\text { to MIPS } 24 \text { for } \sim 6000 \text { galaxies up } \\
\text { to } \mathrm{z}=1 \text {. Consider } 25 \text { different galaxy } \\
\text { types including AGN galaxies. }\end{array}$ \\
\hline
\end{tabular}

Table 1. Classification and comparison of the main characteristics of recent EBL modelings.

a complete understanding of galaxy evolution. Approaches of type (ii) are potentially problematic because they imply extrapolations backwards in time of local or low-redshift luminosity functions (LFs). Intrinsically different galaxy populations exist at high redshifts, which cannot be accounted for by these extrapolations. In particular, Franceschini et al. (2008) use observed LFs in the near-IR from the local Universe to $z=1.4$ to describe the elliptical and spiral populations, and only local information to describe irregular/starbursting galaxies. They distinguish between galaxy morphologies using images from different instruments. Different local LFs and data sets in the IR are used to constrain the mid- and far-IR background. Their modelling is complex and not reproducible. Despite these problems, this methodology is based upon LFs, which are directly observed and well understood unlike type (iii) models based on parameterizations of the history of the SFR density of the Universe, a quantity with large uncertainties and biases.

One important application of the EBL for $\gamma$-ray astronomy is to recover the unattenuated spectra of extragalactic sources. This will not be discussed in this proceedings paper but we refer the interested reader to Domínguez et al. 2011a,b for a discussion about this.

\section{Methodology}

Our model is based on the rest-frame $K$-band galaxy LF from Cirasuolo et al. (2010) and on multiwavelength galaxy data from the All-wavelength Extended Groth Strip International Survey (AEGIS†, Davis et al. 2007) of about 6000 galaxies in the redshift

$\dagger$ http://aegis.ucolick.org/ 
range $0.2-1$. The Cirasuolo et al. (2010) LF is used to count galaxies (and therefore to normalize the total EBL spectral intensity) at each redshift. The LF as well as our galaxy sample is divided into three magnitude bins according to the absolute rest-frame $K$-band magnitude, i.e., faint, middle and bright. Within every magnitude bin, an SED type is statistically attached to each galaxy in the LF assuming SED-type fractions that are a function of redshift within those magnitude bins. This is estimated by fitting our AEGIS galaxy sample to the 25 galaxy-SED templates from the SWIRE $\ddagger$ library (Polletta et al. 2007). Then, luminosity densities are calculated from these magnitude bins from every galaxy population at all wavelengths, and finally all the light at all redshifts is added up to get the overall EBL spectrum.

\section{Results and conclusions}

Fig. 1 shows the local EBL, with its uncertainties, compared with direct and indirect observational data, and other EBL models. Other quantities such as EBL evolution $\dagger$ are discussed in Domínguez et al. (2011a). Fig. 1 suggests that the EBL coming from galaxies is already well constrained in the region from the UV up to the mid-IR but not in the far-IR. The EBL measurements free of zodiacal light in two optical bands by Matsuoka et al. (2011) agree with our EBL estimations. Furthermore, galaxy counts from very deep surveys taken with very sensitive instruments (Madau \& Pozzetti 2000; Fazio et al. 2004; Keenan et al. 2010) should be considered as a good estimation of the true EBL from galaxies. On the other hand, different fully independent modelings based on different methodologies and galaxy data sets such as Franceschini et al. (2008), Gilmore et al. (2011), Domínguez et al. (2011a) agree in the specific intensity level of the EBL. In particular, galaxy count data are in excellent agreement with our EBL estimations. From these results, a relevant contribution from Pop III stars to the local EBL seems unlikely.

Summarizing, the best available data sets are used in our modeling (Cirasuolo et al. 2010's LF and the AEGIS galaxy catalogue) observed over a wide redshift range. This model has the following main advantages over other existing EBL models: transparent methodology, reproducibility, and -very important- utilizing direct galaxy data. The galaxy evolution is directly observed in the rest-frame $K$ band up to $z=4$. Observed galaxies up to $z=1$ from the UV up to $24 \mu \mathrm{m}$ with SEDs of 25 different types (from quiescent to rapidly star-forming galaxies and including AGN galaxies) are taken into account in the same observational framework. A study of the uncertainties in the model (such as uncertainties in the Schechter parameters of the Cirasuolo et al. (2010) LF and the errors in the photometric catalogue) is made directly from the data.

It is concluded that the EBL from galaxies seems already well constrained from UV to mid-IR wavelengths, even though uncertainties are still large in the far-IR. Furthermore, discoveries of $\gamma$-rays from distant blazars (e.g., Aleksic et al. 2011a,b,c) support the EBL specific intensity level derived from galaxy count and recent EBL models such as Gilmore et al. (2011), Franceschini et al. (2008), and Domínguez et al. (2011a). We highlight that the EBL specific intensity calculated with our method matches lower limits from galaxy counts, which implies the highest transparency of the Universe to $\gamma$-ray allowed by standard physics (see Domínguez et al. 2011c). This predicts a promising future for the new generation of imaging atmospheric Cherenkov telescopes, namely CTA.

$\ddagger$ http://www.iasf-milano.inaf.it/ p polletta/templates/swire_templates.html

$\dagger$ EBL specific intensities are publicly available at http://side.iaa.es/EBL 


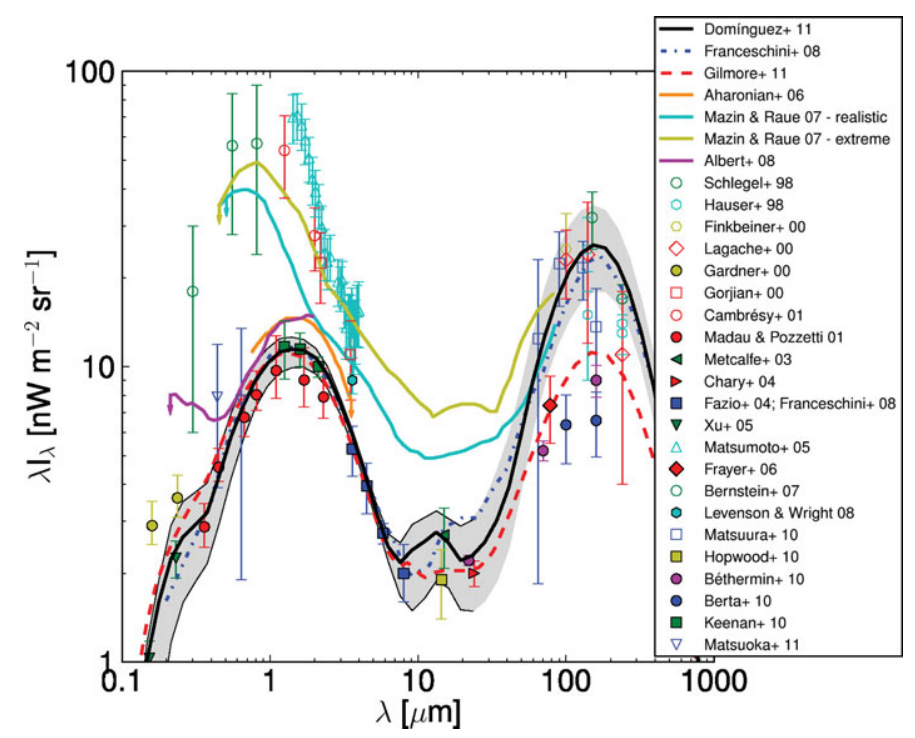

Figure 1. The solid-black line is the extragalactic background light calculated from our methodology. Direct data, data from galaxy count, upper limits from $\gamma$-ray astronomy and other recent EBL modelings are shown as well (see Domínguez et al. 2011a for details).

\section{Acknowledgements}

I am grateful for financial support from a Fermi grant to participate in the IAU 284 Symposium on The Spectral Energy Distribution of Galaxies.

\section{References}

Aleksić, J., et al. 2011a, ApJ, 726, 58

Aleksić, J., et al. 2011b, ApJ, 730, L8

Aleksić, J., et al. 2011c, $A \& A$ A, 530, A4

Bruzual, A. G. \& Charlot, S. 1993, ApJ, 405, 538

Bruzual, A. G. \& Charlot, S. 2003, MNRAS, 344, 1000

Charlot, S. \& Fall, S. M. 2000, ApJ, 539, 718

Chary, R.-R. \& Pope, A. 2010, arXiv:1003.1731

Cirasuolo, M., et al. 2010, MNRAS, 401, 1166

Davis, M., et al. 2007, ApJ, 660, L1

Domínguez A., et al. 2011a, MNRAS, 410, 2556

Domínguez A., et al. 2011b, Proceeding of the 25th Texas Symposium, arXiv:1103.4534

Domínguez, A., Sánchez-Conde, M. A., \& Prada, F. 2011c, JCAP (in press), arXiv:1106.1860

Fazio, G. G., et al. 2004, ApJS, 154, 39

Finke, J. D., Razzaque, S., \& Dermer, C. D. 2010, ApJ, 712, 238

Franceschini, A., Rodighiero, G., \& Vaccari, M. 2008, A\&A, 487, 837

Gilmore, R. C., Somerville, R. S., Primack, J. R., \& Domínguez, A. 2011, arXiv:1104.0671

Hauser, M. G. \& Dwek, E. 2001, ARA\&A, 39, 249

Keenan, R. C., Barger, A. J., Cowie, L. L., \& Wang, W.-H. 2010, ApJ, 723, 40

Kneiske, T. M. \& Dole, H. 2010, A\&A, 515, A19

Matsuoka, Y., Ienaka, N., Kawara, K., \& Oyabu, S. 2011, ApJ, 736, 119

Madau, P. \& Pozzetti, L. 2000, MNRAS, 312, L9

Polletta, M., et al. 2007, ApJ, 663, 81

Rieke, G. H., et al. 2009, ApJ, 692, 556

Somerville, R. S., Gilmore, R. C., Primack, J. R., \& Domínguez, A. 2011, arXiv:1104.0669 